\title{
Product Construction of Key Distribution Schemes for Sensor Networks
}

\author{
Reizhong Wei and Jiang Wu \\ Department of Computer Science, Lakehead University, \\ Thunder Bay, Ontario P7B 5E1, Canada \\ wei@ccc.cs.lakeheadu.ca \\ jwu1@lakeheadu.ca
}

\begin{abstract}
Wireless sensor networks are composed of a large number of randomly deployed sensor nodes with limited computing ability and memory space. These characteristics give rise to much challenge to key agreement. General key agreement schemes like KDC, PKI and the DiffieHellman key exchange schemes are not applicable to sensor networks. Recently several key distribution schemes have been proposed specifically for sensor networks, aimed to provide high connectivity and resilience while keeping low memory usage in the sensor nodes. In this paper, we formularize and analyze these methods, and deduce general conditions for a scheme to be optimal in terms of connectivity, resilience and memory usage. The result provides guideline to design optimal schemes. Based on the result, we proposed 2 schemes that can achieve optimal connectivity and resilience with the restriction of memory space.
\end{abstract}

\section{Introduction}

A distributed sensor network is composed of a large number of sensor nodes that are densely deployed. The position of sensor nodes usually are not predetermined. This allows random deployment in inaccessible terrains or disaster relief operations. This means that sensor network protocols and algorithms must possess self-organizing capabilities. In general, a sensor node is battery powered and equipped with integrated sensors, data processing capabilities, and short-range radio communications. Examples of sensor network protocols include SmartDust [9] and WINS 1]. There is a wide range of applications for sensor networks. Some examples of the application areas are health, military, and smart environment (see, e.g., 2]).

To secure communications for a sensor network is extremely important, as the network is prone to different types of malicious attacks when it is deployed in a hostile environment. An adversary can compromise sensor nodes much easier than to compromise computers. However, since the limitation in both the memory resources and computing capacity of a sensor node, it is impractical to use public-key cryptosystems to secure sensor networks. Using a traditional Internet style key exchange and key distribution protocols based on trusted third parties are also impractical. 
To solve the key management problem for sensor networks, several researchers considered special key pre-distribution schemes. In [7, Eschnauer and Gligor used random methods to distribute keys. In their scheme, each sensor node received a random subset of keys from a large key pool before deployment. Any two nodes able to find one common key within their respective subsets can use that key as their shared secret to initiate communication. Some theory of random graphs was used to analyze their scheme. Based on this scheme, Chan, Perrig and Song in 4] proposed a $q$-composite random key pre-distribution scheme. In their scheme, $q$ common key instead of just one common key are used to establish secure communications between two nodes, which increases the security (resilience) of the network. Recently, Du, Deng, Han and Varshney in [6] and Liu and Ning in 10] used a new method to construct key distribution schemes, which we will call it product construction. In their method, they combined traditional pairwise key distribution scheme with other schemes to construct new key distribution schemes. Their methods improved network resilience comparing to previous key pre-distribution schemes. The purpose of this paper is to formularize and analyze their methods in order to optimize this method. Upon these analysis, some combinatorial methods are then used to improve their constructions.

When we design a key distribution scheme for a distributed sensor network, the following key characteristics of the design must be considered.

- Small key size: Since the limited resource of a sensor node, key storage should be small. For example, if there are $b$ nodes in the network, then we cannot expect that a node can store $b-1$ keys to share a secrete key with each of the other nodes.

- Resilience of the network: Even quite a large amount of sensor nodes are compromised by an adversary, the communications among other nodes should be still secure. In other words, a coalition of certain number of sensor nodes cannot compute other secrete keys used by other sensor nodes.

- Local connectivity: A sensor node should be able to securely communicate with its local neighbors. Here a local neighbor means a sensor node physically located within transmission range.

- Global connectivity: Any two nodes of the sensor network are connected. So for any two nodes $u$ and $v$ in the network, there are notes $c_{1}, c_{2} \cdots c_{t}$ such that $u$ and $c_{1}$ share a secret key, $c_{i}$ and $c_{i+1}$ share a secret key for $i=1, \cdots, t-1$ and $c_{t}$ and $v$ share a secret key.

We will only consider schemes satisfying all these properties. The main contributions of this paper are as follows. First we use a uniformed method to generalize the methods used in 6, 10. We define a product of a key distribution scheme and a set system and use that definition to construct new key distribution schemes. Then we use combinatorial methods to analyze the product construction and give some necessary conditions to optimize the product construction. Finally, we propose new constructions which meet all of these necessary conditions.

The rest of this paper is organized as follows. Section 2 defines production construction. In Section 3, set system used in the production construction is 
analyzed using combinatorial methods. Section 4 describes our proposed schemes which is then compared with the previous schemes. Section 5 concludes the paper.

\section{Product Construction}

In this section, we give a generalized description of the schemes in [6] and [10, which used a similar method to construct key pre-distribution schemes for sensor networks. We start with a definition of a pairwise key pre-distribution scheme.

Definition 1. A pairwise key pre-distribution scheme $D$ is a triple $(\mathcal{U}, \mathcal{F}, \mathcal{K})$, where $\mathcal{U}$ is a set of nodes, $\mathcal{F}$ is a set of algorithms and $\mathcal{K}$ is a set of keys, which satisfies the following conditions:

1. For each $u \in \mathcal{U}$ an $f_{u} \in \mathcal{F}$ is assigned to $u$;

2. For any $u, v \in \mathcal{U}$ there is a unique key $K_{u, v} \in \mathcal{K}$ shared between $u$ and $v$, which can be obtained from $f_{u}$ and from $f_{v}$;

3. For any other $w \in \mathcal{U}$, no information about $K_{u, v}$ can be obtained by $f_{w}$.

The above definition shows that we are considering unconditional secure schemes (not for computational secure ones).

If a pairwise key pre-distribution scheme has the property that even $\lambda$ nodes are compromised the system is still secure, then we say that the scheme is $\lambda$ secure, or the scheme is $\lambda$ resilience. More formally, in a $\lambda$-secure pairwise key pre-distribution scheme, for any $w_{1}, w_{2}, \cdots, w_{\lambda} \in \mathcal{U}, K_{u, v}$ cannot be computed by $f_{w_{1}}, f_{w_{2}}, \cdots, f_{w_{\lambda}}$ where $u, v$ are different from $w_{1}, w_{2} \cdots, w_{\lambda}$.

Note that it is not necessary to use a pairwise key pre-distribution scheme to a sensor network, because even two nodes shared a common key, they may not be able to communicate each other when their distance is beyond transmission range. For a sensor network, local communications are more important. So we will consider both the local connectivity and the global connectivity of a key distribution scheme for our purpose.

An example of $\lambda$-secure key pre-distribution scheme is the Blom's scheme [3] in which each node stores $\lambda+1$ keys.

The Blom's scheme can be described as follows. Suppose there are $b$ nodes $u_{1}, u_{2}, \cdots, u_{b}$ in a network. To distribute keys, an authorized center (AC) chooses a random bivariate symmetric polynomial in a finite field $G F(q)$ :

$$
f(x, y)=\sum_{i=0}^{\lambda} \sum_{j=0}^{\lambda} a_{i, j} x^{i} y^{j},
$$

where $a_{i, j}=a_{j, i}$. Then the CA gives $P_{i}(x)=f(x, i)$ to $u_{i}$ as its personal key. The common key between $u_{i}$ and $u_{j}$ is $P_{i}(j)=P_{j}(i)=f(i, j)$. It is proved using a linear algebra method that a Blom's scheme is $\lambda$-secure.

To formularize the methods used in [6, 10, we need some concepts from combinatorics which we introduce below. 
A set system $S$ is a pair $(X, \mathcal{B})$ where $X$ is a set of points and $\mathcal{B}$ is a collection of $k$-subsets (called blocks) of $X$. For our purpose, same blocks are allowed in a set system.

Suppose there is a map from the set of nodes $\mathcal{U}$ to the set of blocks $\mathcal{B}$ of a set system so that for a $u_{i} \in \mathcal{U}$ there is a unique $B_{i} \in \mathcal{B}$ corresponding to it. Then we can define a product of $D$ and $S$ as follows.

Definition 2. Suppose $D=(\mathcal{U}, \mathcal{F}, \mathcal{K})$ is a pairwise key pre-distribution scheme and $S=(X, \mathcal{B})$ is a set system, where $|\mathcal{B}| \geq|\mathcal{U}|$. Suppose there is also a map from $\mathcal{U}$ to $\mathcal{B}$ such that a $u_{i} \in \mathcal{U}$ is mapped to a $B_{i} \in \mathcal{B}$. A product of $D$ and $S$, $D \times S$, is defined as a triple $(\mathcal{U}, \mathcal{F} \times \mathcal{B}, \mathcal{K} \times X)$ such that the algorithm assigned to $u_{i}$ is $f_{u_{i}} \times B_{i}$.

The method used in [6] and [10] for key establishment of sensor networks actually is the above product method. Both of the papers used Blom's scheme as $D$. 10 proposed two set systems. One is random subset assignment. In this assignment, each node gains a random $\tau$-subset of $X$, so $\mathcal{B}$ contains $u$ random $\tau$-subsets (by this setting, repeated blocks are allowed). The other proposed set system in [10] is grid-based system. In this system, $X=M_{1} \cup M_{2} \cup \cdots \cup M_{t}$, where $M_{1}, M_{2}, \cdots M_{t}$ are disjoint $m$-sets for $m \geq u^{1 / t}$. The set $\mathcal{B}$ contains all the blocks from $\left\{\left(i_{1}, i_{2}, \cdots, i_{t}\right): i_{1} \in M_{1}, \cdots, i_{t} \in M_{t}\right\}$. [6] used the random subset assignment.

As an example, in the following we give a brief description of the random subset assignment used in both [6] and 10.

To distribute keys, the AC chooses a set $X$ where $|X|=v$. Then for each element $i \in X$, the $\mathrm{AC}$ generates a random symmetric polynomial $f_{i}(x, y)$ as in a Blom's scheme. So $v$ polynomials are generated. For a node $u_{j} \in \mathcal{U}$, the $\mathrm{AC}$ chooses a random $k$-subset of $X$. Denote the subset as $B=\left\{i_{1}, i_{2}, \cdots, i_{k}\right\}$. The keys given to $u_{j}$ are $f_{i_{s}}(x, j)$, for $1 \leq s \leq k$. The choice of $v$ and $k$ depends on the connectivity of the network. To form a secret key between $u_{i}$ and $u_{j}$, they will try to find a common element in the subset assigned to them. If they found the element, say $i_{0}$, then the secret key is $f_{i_{0}}(i, j)$.

Sometimes we also can view the product construction as using different copies of a pairwise key distribution scheme $D$ and denote it as $D \times X$. So $(D, i)$ will be used to denote the $i$ th scheme. It's also called a key space in this paper.

The main purpose of using a set system is to add resilience of the key distribution scheme. However, 6] and [10] only discussed the specific set systems used in their schemes. In next section, we will discuss how a set system effects the resilience and connectivity of the product scheme in general.

\section{Analysis of the Set System}

For a better key pre-distribution system, we should consider several things: the resilience of the system, the storage space requested for a node, the connectivity of the network, etc. Basically, the storage space requested for a node depends on the size of a block in the set system. In this section, we discuss how the set 
system used in the product construction effects the resilience and connectivity of the network if the size of block is fixed.

\subsection{Resilience}

Suppose the resilience of the original key pre-distribution scheme $D$ is $\lambda$. When a set system $S$ is used in the product construction, we need to consider the probability that one of the schemes in the product system $D \times S$ is broken. For example we consider the probability that $(D, 1)$ is broken (We denote this event as $\left.D_{1}\right)$. Let $p_{j}^{1}$ denote the probability that exact $j$ blocks out of $s$ blocks contain 1. Let $C_{s}$ denote the event that $s$ nodes were compromised. Then we have

$$
\operatorname{Pr}\left(D_{1} \mid C_{s}\right)=\sum_{j=\lambda+1}^{s} p_{j}^{1} .
$$

Therefore we want to keep $p_{j}^{1}$ as small as possible. On the other hand, since $D_{i}$ and $D_{j}$ are independent and we want the probability that any space is broken as small as possible, we have the following result about the structure of $D$, which gives some necessary condition for $D$.

Theorem 1. In a product scheme $D \times S$, suppose $D$, the size of $X$, the size of $\mathcal{B}$ and the size of a block are fixed. Then each element of $X$ should appear in equal number of blocks to keep the optimal resilience of the scheme.

Proof. Suppose all the parameters of the set system mentioned in the theorem are fixed. Let $b=|\mathcal{B}|, k$ be the size of a block. Suppose $X=\{1,2, \cdots, v\}$ and $i \in X$ appears in $r_{i}$ blocks. Then the probability that exact $j$ out of $s$ blocks contain $i$ is

$$
p_{j}^{i}=\frac{\left(\begin{array}{c}
r_{i} \\
j
\end{array}\right)\left(\begin{array}{c}
b-r_{i} \\
s-j
\end{array}\right)}{\left(\begin{array}{l}
b \\
s
\end{array}\right)},
$$

which depends on the value of $r_{i}$. Since $\sum_{i=1}^{v} r_{i}=k b$ is fixed, if there are some $i$ such that the value of $\sum_{j=\lambda+1}^{s} p_{j}^{i}$ is small, then there must be some $t$ such that $\sum_{j=\lambda+1}^{s} p_{j}^{t}$ is larger. That means $(D, t)$ is easier to break.

In intuition, if an element in $X$ appears in more blocks, then the corresponding key space is weaker. So we want the elements distributed evenly.

It is easy to check that the grid-based system satisfies the condition of Theorem 1 (However, we will see later that its local connectivity is not good). Theoretically, the random subset assignment also satisfies the condition of Theorem 1 in a sense of probability. However, in practice the random subset assignment may violate that condition. For example the worst case of the random subset assignment will not fit the condition of Theorem 11. So we want some deterministic method to find a set system that has even distributions of elements.

Suppose each element appears in $r$ blocks. Then we have

$$
p_{j}^{i}=\frac{\left(\begin{array}{l}
r \\
j
\end{array}\right)\left(\begin{array}{l}
b-r \\
s-j
\end{array}\right)}{\left(\begin{array}{l}
b \\
s
\end{array}\right)} .
$$

Therefore the value of $\operatorname{Pr}\left(D_{i} \mid C_{s}\right)$ is determined by the values of $r$ and $b$. 
To obtain a set system satisfying the condition of Theorem 1, we need a definition from combinatorial design theory. For general information about combinatorial design theory used in this paper, see [11.

Definition 3. A 1-design $S_{r}(1, k, v)$ is a set system $(X, \mathcal{B})$ such that each element $x \in X$ is contained in exactly $r$ blocks, where $v=|X|, k$ is the block size.

The following construction is from [11-Theorem 9.10].

Theorem 2. There exists an $S_{r}(1, k, v)$ with $b$ blocks if $b=v r / k$ is an integer.

Proof. Let $u=\operatorname{gcd}(k, r)$. Then $r=u r^{\prime}$ and $k=u k^{\prime}$ where $\operatorname{gcd}\left(r^{\prime}, k^{\prime}\right)=1$. Since $b=v r / k=v r^{\prime} / k^{\prime}$ and $\operatorname{gcd}\left(r^{\prime}, k^{\prime}\right)=1$, it must be the case that $v \equiv 0 \quad\left(\bmod k^{\prime}\right)$. Let $v=s k^{\prime}$ where $s$ is a positive integer. Then $b=s r^{\prime}$.

Let $Y$ be a set of cardinality $k^{\prime}$, and define $X=Y \times \mathbb{Z}_{s}$. Let $A_{1}, A_{2}, \cdots, A_{r^{\prime}}$ be $r^{\prime}$ arbitrary $u$-subsets of $\mathbb{Z}_{s}$. For $1 \leq i \leq r^{\prime}$, define $B_{i}=Y \times A_{i}$. Then each $B_{i}$ is a $k$-subset of $X$. Now for each $B_{i}$, we develop $s$ blocks $B_{i}^{j}$ as follows. Suppose $B_{i}=Y \times\left\{s_{1}, s_{2}, \cdots, s_{r^{\prime}}\right\}$. Then for each $j, 1 \leq j \leq s-1$, let $B_{i}^{j}=$ $Y \times\left\{s_{1}^{\prime}, s_{2}^{\prime}, \cdots, s_{r^{\prime}}^{\prime}\right\}$, where $s_{t}^{\prime}=s_{t}+j \quad(\bmod s), 1 \leq t \leq r^{\prime}$. The result is an $S_{r}(1, k, v)$.

When $r=\left(\begin{array}{l}v \\ k\end{array}\right)$, we have a easy way to construct a 1-design.

Theorem 3. There exists an $S_{r}(1, k, v)$ for $r=\left(\begin{array}{l}v-1 \\ k-1\end{array}\right)$ and $b=\left(\begin{array}{l}v \\ k\end{array}\right)$.

Proof. Let the set of blocks contains all the $k$-subsets of a $v$-set.

Suppose we fix $|X|=v$, and the size of block is $k$. Then we want each element belongs to $\frac{k b}{v}$ blocks so that the condition of Theorem 1 is satisfied. In practice, we let $b$ be multiples of $v$ and $r=\frac{k b}{v}$.

\subsection{Connectivity}

To consider the connectivity of a sensor network, we consider the graph $G(\mathcal{U}, E)$, where two nodes are connected by an edge if and only if these two nodes share at least one common secret key. Following from the method used in [7, we view a sensor network as a random graph. Since the connectivity of a random graph is a monotone property (when the number of nodes are fixed, the probability of connectivity is increasing when the number of edges is increasing), according to a theory of [8], the expected node degree $d$ can be computed as follows:

$$
d=\frac{b-1}{b}\left(\ln b-\ln \left(-\ln P_{c}\right)\right)
$$

where $b=|\mathcal{U}|$ and $P_{c}$ is the probability that the random graph is connected. Therefore the connectivity of the network depends on the degree $d$ when the number of nodes are fixed. 
In the product construction, two nodes share a common secret key if and only if their blocks have at least one common element. Suppose in the set system, each block intersects $t$ other blocks. For a given density of sensor network deployment, if the expected value of number of neighbors is $n$, then $d=\frac{n t}{b}$. So we have the following result.

Lemma 1. The connectivity of the product scheme depends on the number of blocks which share at least one element with a block in the set system.

From Lemma 1, we know that the connectivity of the scheme from grid-based system in [10] is not good. In that system, each block intersects $t v^{1 / t}-t$ other blocks. However, we will see later that using other set system will improve the connectivity of the network a lot.

Suppose $X=\{1,2, \cdots, v\}$ and $\mathcal{B}=\left\{B_{1}, B_{2}, \cdots, B_{b}\right\}$. An incidence matrix of the set system $(X, \mathcal{B})$ is a $b \times v 0-1$ matrix $A=\left(a_{i, j}\right)$, where

$$
a_{i, j}= \begin{cases}1 & \text { if } j \in B_{i} \\ 0 & \text { otherwise }\end{cases}
$$

Let $C=A A^{T}=\left(c_{i, j}\right)$. Then $C$ is a symmetric $b \times b$ matrix. Suppose each element of $X$ appears in $r$ blocks. Then we have $c_{i, i}=k, 0 \leq c_{i, j} \leq k$ and

$$
\sum_{i=1, i \neq j}^{b} c_{i, j}=k(r-1) .
$$

The number of blocks which intersect block $B_{i}$ equals to the number of nonzero elements in the $i$ th row of $C$. So if we want to keep the local connectivity as large as possible, we need to let the number of nonzero elements in $C$ as large as possible. In other words, we want to keep the individual $c_{i, j}$ as small as possible. In intuition, we don't want repeat blocks to avoid the case that $c_{i, j}=k$ for some $i \neq j$.

Remark 1. From (2) and (3) we can see that there is a trade-off between the resilience and connectivity of the network. For the connectivity, we want $r$ to be large. However, when $r$ is larger the probability that a scheme is broken is increasing.

The following result indicates that the construction of Theorem 3 is optimal.

Theorem 4. When $b=\left(\begin{array}{l}v \\ k\end{array}\right), r=\left(\begin{array}{l}v-1 \\ k-1\end{array}\right)$ and $v>2 k$, the set system constructed in Theorem 3 has the largest number of intersections for a block.

Proof. It is easy to know that a block intersects

$$
I=\left(\begin{array}{l}
v \\
k
\end{array}\right)-\left(\begin{array}{c}
v-k \\
k
\end{array}\right)-1
$$

other blocks in the set system of Theorem 3 . We are going to prove that if there are repeated blocks in an $S_{r}(1, v, k)$, then a block intersects less blocks. 
Suppose there are two identical blocks. Then each element in that block appears in $r-2$ other blocks. So that block can intersect at most $I^{\prime}=k(r-2)+1$ other blocks. Since $\left(\begin{array}{l}v \\ k\end{array}\right)=\frac{v}{k}\left(\begin{array}{l}v-1 \\ k-1\end{array}\right)$ and $r=\left(\begin{array}{l}v-1 \\ k-1\end{array}\right)$ we have

$$
I=\frac{v}{k}\left(\begin{array}{c}
v-1 \\
k-1
\end{array}\right)-\left(\begin{array}{c}
v-k \\
k
\end{array}\right)-1,
$$

and

$$
\begin{aligned}
I-I^{\prime} & =\frac{v-k}{k}\left(\begin{array}{c}
v-1 \\
k-1
\end{array}\right)-\left(\begin{array}{c}
v-k \\
k
\end{array}\right)+2 k-2 \\
& =\frac{(v-1)(v-2) \cdots(v-k)}{(v-k)(v-k-1) \cdots(v-2 k+1)}\left(\begin{array}{c}
v-k \\
k
\end{array}\right)-\left(\begin{array}{c}
v-k \\
k
\end{array}\right)+2 k-2 \\
& =\left(\frac{(v-1)(v-2) \cdots(v-k)}{(v-k)(v-k-1) \cdots(v-2 k+1)}-1\right)\left(\begin{array}{c}
v-k \\
k
\end{array}\right)+2 k-2 \\
& >0
\end{aligned}
$$

The conclusion follows.

In order to use construction of Theorem 2 we need to consider how to choose the sets $A_{1}, A_{2}, \cdots, A_{r^{\prime}}$. Suppose $S \subseteq \mathbb{Z}_{s}$, where $\mathbb{Z}_{S}$ is the additive group of order $s$. Define the differences of $S$ as:

$$
\nabla S=\left\{x-x^{\prime} \quad(\bmod s): x, x^{\prime} \in S, x \neq x^{\prime}\right\} .
$$

If an element of $\mathbb{Z}_{s} \backslash\{0\}$ appears $t$ times in $\cup_{i} \nabla S_{i}$ for some subsets $S_{i}$, then we say that the element has $t-1$ repeatings. The sum of the repeatings of all elements is called the repeatings of $\cup_{i} \nabla S_{i}$.

Theorem 5. The 1-design constructed from Theorem 2 has the largest local connectivity, if the collection

$$
\cup_{i=1}^{r^{\prime}} \nabla\left(A_{i}\right)
$$

contains least repeatings.

Proof. If an element $g \in \mathbb{Z}_{s} \backslash\{0\}$ has $t-1$ repeatings in $\cup_{i=1}^{r^{\prime}} \nabla\left(A_{i}\right)$, then pairs $\left(x_{i}, x_{j}\right)$ appear in $t$ blocks, where $x_{i}-x_{j}=g$. Since each element appears in $r$ blocks, we want to reduce the number of blocks containing a same pair of elements to maximize the number of blocks which intersect a fixed block.

Definition 4. Let $G$ be an additive abelian group of order $v$. A set system $(G, \mathcal{B})$ is called $a(v, k, \lambda)$ difference family if every nonzero element of $G$ occurs $\lambda$ times in

$$
\cup_{B \in \mathcal{B}} \nabla B
$$

For example, a $(13,3,1)$ difference family contains blocks $\{0,1,4\}$ and $\{0,2,7\}$. There are many results about the construction of difference families in literature (see i.e., [5]). From Theorem [5] we know that we can use blocks in a $(v, k, \lambda)$ difference family with smallest $\lambda$ to construct 1-design and then obtain a good product scheme. 


\section{Proposed Schemes}

Several recently proposed key distribution schemes used random distribution method [4, 6, 7, 10]. There are reasons that deterministic method should be developed as well. For example, the theoretical analysis shows scheme in 6] can provides good connectivity, resilience and memory consuming attributes to sensor networks. But the random distribution of the keys leaves open issues in practical implementation. We can view this scheme as a $D \times S$ scheme. Here $S$ is a set system $(X, B)$, where $X=\left\{A_{1}, A_{2}, \cdots, A_{v}\right\}, B=\left\{B_{1}, B_{2}, \cdots, B_{b}\right\}$, and each $B_{i}$ contains $k$ elements randomly selected from $X$. As we analyzed in Section 3, in a sense of probability this scheme meets Theorem 1 and 5 to achieve optimal connectivity and resilience. But in real implementation, it may produce worse result. The result also depends on the random number generating function used to generate the $S$ set system. Different random number generators may result in different $S$ systems. It is necessary to design a deterministic distribution scheme that always meets Theorem 1 and 5 for the purpose of real applications.

In this section we consider the construction of set systems used in the key distribution scheme. Given the scale and connectivity of the sensor networks, the memory space of each sensor node, we need to determine which set system to be used, what are the parameters of the selected set system, and how to construct the set system.

The predefined requirements and restriction on the sensor network include:

$-b$, the number of nodes in the sensor network,

- $M$, the memory space of a sensor node to store the keys,

$-P_{c}$, the probability that the random graph of the sensor nodes are connected, and

- $n$, the estimated number of neighbors of a sensor node after deployment.

The constructed set systems should meet the above requirements and restrictions and at the same time achieve optimal resilience. Analysis in Section 3 shows that set systems that meet Theorem 1 and 5 can obtain optimal resilience and connectivity. In the next parts we give construction using 2 such set systems.

\subsection{Construction with $(v, k, 1)$ Difference Families}

From Definition 4 we know that $(v, k, 1)$ Difference Families meet conditions of Theorem 1 and 5. In this subsection, we give the construction using $(v, k, 1)$ Difference Families, and analyze its performance.

First we compute $P_{\text {connect }}$, which is the probability that a pair of nodes share at least one common key space:

$$
P_{\text {connect }}=\frac{b-1}{n b}\left(\ln b-\ln \left(-\ln P_{c}\right)\right) .
$$

Next we choose a proper $(v, k, 1)$ difference family that can provide the desired connectivity. We compute $P_{v k 1}$, the probability that a pair of blocks from the $(v, k, 1)$ shares an element: 


$$
P_{v k 1}=\frac{k^{2}\left(r^{\prime}-1\right)+k(k-1)}{v r^{\prime}-1},
$$

where $r^{\prime}$ is the number of basic blocks of the chosen family. Also we can compute the number of blocks of the $(v, k, 1)$ :

$$
r=k r^{\prime}
$$

The following table chooses a sample collection of $(v, k, 1)$ difference families from [5] and computes their parameters:

\begin{tabular}{|c|c|c|c|c|}
\hline $\mathrm{v}$ & $\mathrm{k}$ & $\mathrm{r}^{\prime}$ & $\mathrm{r}$ & $P_{v k 1}$ \\
\hline 25 & 3 & 4 & 100 & 0.33 \\
27 & 3 & 5 & 135 & 0.31 \\
31 & 3 & 5 & 155 & 0.27 \\
33 & 3 & 6 & 198 & 0.25 \\
37 & 3 & 6 & 222 & 0.23 \\
39 & 3 & 7 & 273 & 0.22 \\
43 & 3 & 7 & 301 & 0.20 \\
40 & 4 & 4 & 160 & 0.38 \\
49 & 4 & 6 & 196 & 0.31 \\
\hline
\end{tabular}

Usually there are more than one families whose connectivity are better than and close to $P_{\text {connect }}$. We choose the one with least blocks that is larger than $b$, and assign each block to a sensor node.

The third step is to construct $v$ key spaces. We computer the security threshold $\lambda$ of the key space:

$$
\lambda=\left\lfloor\frac{M}{k}\right\rfloor-1
$$

The construction of the key spaces is the same as that in [6] which is equivalent to a Blom's scheme. We briefly introduce an example as follows.

1. Select a primitive element $s$ from a finite field $G F(q)$, where $q$ is the least prime larger than the key size, then generate the following $(\lambda+1) \times b$ matrix $G$ :

$$
G=\left[\begin{array}{ccccc}
1 & 1 & 1 & 1 & 1 \\
s & s^{2} & s^{3} & \cdots & s^{b} \\
s^{2} & \left(s^{2}\right)^{2} & \left(s^{3}\right)^{2} & \cdots & \left(s^{b}\right)^{2} \\
\vdots & \vdots & \vdots & \cdots & \vdots \\
s^{\lambda} & \left(s^{2}\right)^{\lambda} & \left(s^{3}\right)^{\lambda} & \cdots & \left(s^{b}\right)^{\lambda}
\end{array}\right]
$$

2. Randomly generate $v$ symmetric matrix $D_{1}, \ldots, D_{v}$ of size $(\lambda+1) \times(\lambda+1)$, then compute the matrixes $A_{i}=\left(D_{i} \cdot G\right)^{T}$, for $1 \leq i \leq v$. Here we get $v$ key spaces $A_{1}, \cdots, A_{v}$. Every key space is $\lambda$-secure. 
Finally, the key spaces are assigned to the sensor nodes according to the blocks. For example, if a block $\{2,3,4\}$ was assigned to node 5 , the 5 th rows of matrixes $A_{2}, A_{3}$ and $A_{4}$ are assigned to node 5 .

We give an illustration of the resilience of the scheme using $(v, k, 1)$ (we call it $(v, k, 1)$ scheme). We use the $P_{\text {break }}=\operatorname{Pr}\left(D_{1} \mid C_{s}\right)$ defined in (11) as an indication of resilience, and plot it as a function of number of compromised node in Figure 1. In the figure, $M$ is set to 200, 3 schemes with different parameters and connectivity are shown. We see that to achieve a probability of 0.5 to break 1

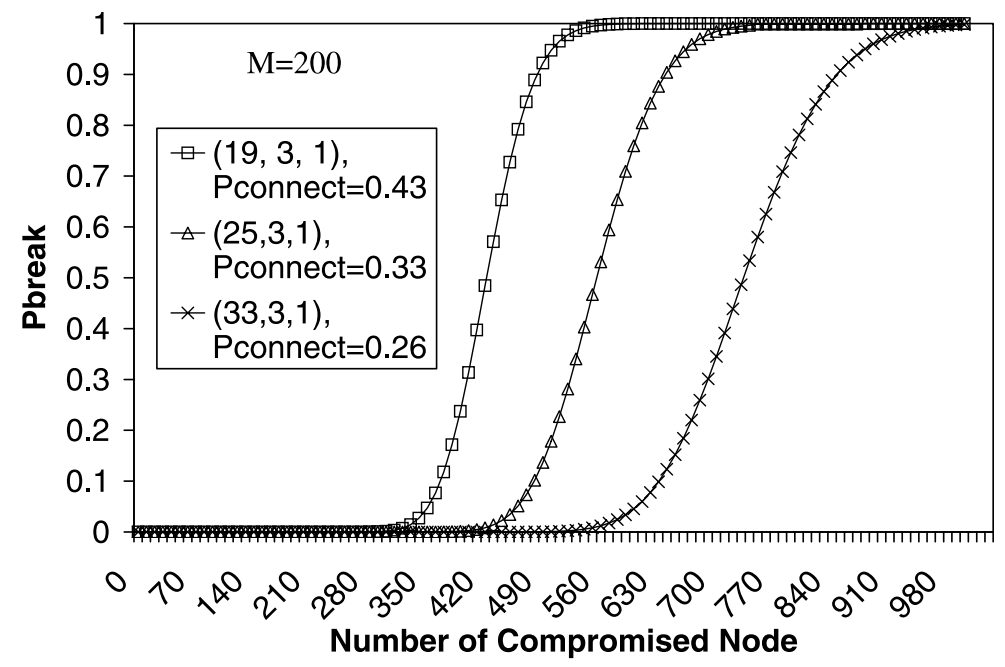

Fig. 1. Probability that one key space is broken

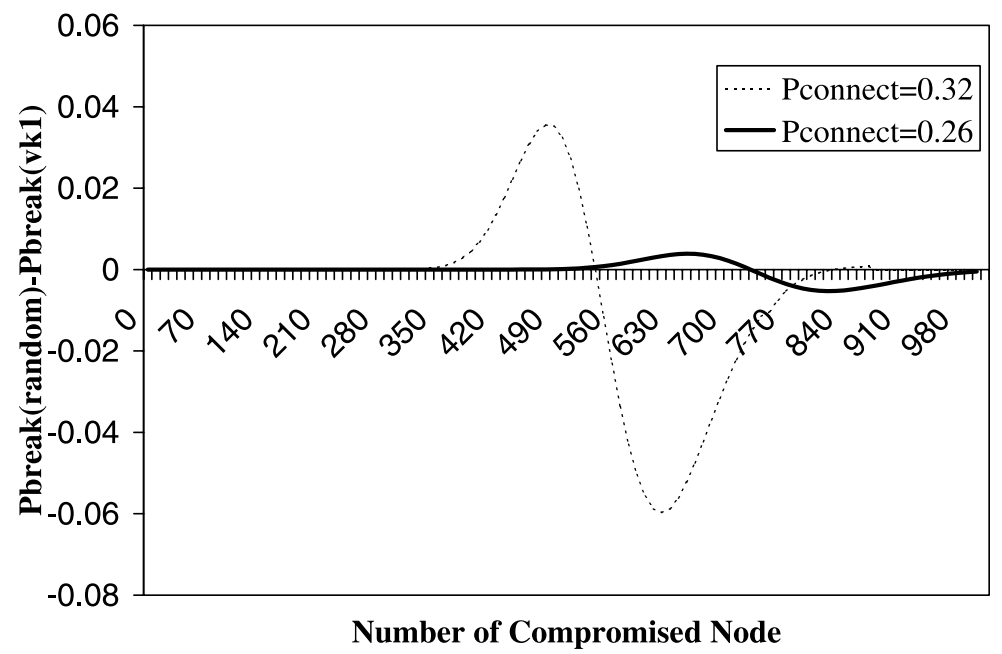

Fig. 2. Difference between $P_{b r e a k}$ of $(v, k, 1)$ scheme and random scheme 
key space, more than 200 nodes are to be compromised. The lower the $P_{\text {connect }}$ of the $(v, k, 1)$, the more compromised nodes are needed. This is the same attribute that the scheme in [6] (we call it random scheme in the following parts) provides.

Now we compare the $(v, k, 1)$ scheme with the random scheme in Figure 2 Figure 2 shows the difference between $P_{\text {break }}$ of pairs of $(v, k, 1)$ and random schemes with same $M$ and similar $P_{\text {connect }}$. The figure shows the difference is very small, and generally, the 2 schemes risk similar compromise possibility.

\subsection{Construction Using all $k$-Subsets}

One potential drawback of the $(v, k, 1)$ scheme is that its number of blocks is limited. So when the network size is large, we consider using all $k$-subsets which provides large block size easily. As proved in Theorem 3 and 4 , the set system of all $k$-subsets meets Theorem 1 and 5 and can provide optimal connectivity and resilience. We give construction steps using that set system as follows.

First, we compute $P_{\text {connect }}$ using (4), then we need to find the $v$ and $k$ so that the set system meets the requirement on the scale and connectivity. The next 2 conditions need to be met:

$$
\begin{aligned}
P_{\text {connect }} & \geq 1-\frac{\left(\begin{array}{c}
n-k \\
k
\end{array}\right)}{\left(\begin{array}{c}
n \\
k
\end{array}\right)-1} \\
b & \leq\left(\begin{array}{l}
v \\
k
\end{array}\right)
\end{aligned}
$$

The above functions produce a list of tables for $v, k, P_{\text {connect }}$ and $b$. From the tables, given $P_{\text {connect }}$ and $b$, we can get corresponding $v$ and $k$. Following is a sample table for Pconnect $=0.3$ :

\begin{tabular}{|c|c|c|}
\hline $\mathrm{v}$ & $\mathrm{k}$ & $\mathrm{b}$ \\
\hline 20 & 3 & 1140 \\
21 & 3 & 1330 \\
22 & 3 & 1540 \\
23 & 3 & 1771 \\
\hline
\end{tabular}

With $v$ and $k$, it is easy to construct the set system of all $k$-subsets.

For the all $k$-subsets scheme, the resilience is

$$
P_{\text {break }}=\sum_{j=\lambda+1}^{s} \frac{\left(\begin{array}{l}
d \\
j
\end{array}\right)\left(\begin{array}{l}
x-d \\
s-j
\end{array}\right)}{\left(\begin{array}{l}
x \\
s
\end{array}\right)}
$$

where $x=\left(\begin{array}{l}v \\ k\end{array}\right), d=\left(\begin{array}{l}v-1 \\ k-1\end{array}\right)$ and $\lambda=\left\lfloor\frac{M}{k}\right\rfloor-1$.

The $P_{\text {connect }}$ of all $k$-subsets scheme is

$$
P_{\text {connect }}=1-\frac{\left(\begin{array}{c}
v-k \\
k
\end{array}\right)}{\left(\begin{array}{l}
v \\
k
\end{array}\right)-1},
$$

which is very close to that of random scheme. 
We compare the all $k$-subsets scheme with random scheme with the same $v$ and $k$ in Figure 3. $M$ is set to 200 here. The figure shows the difference between $P_{\text {break }}$ of all $k$-subsets and random sets is very small.

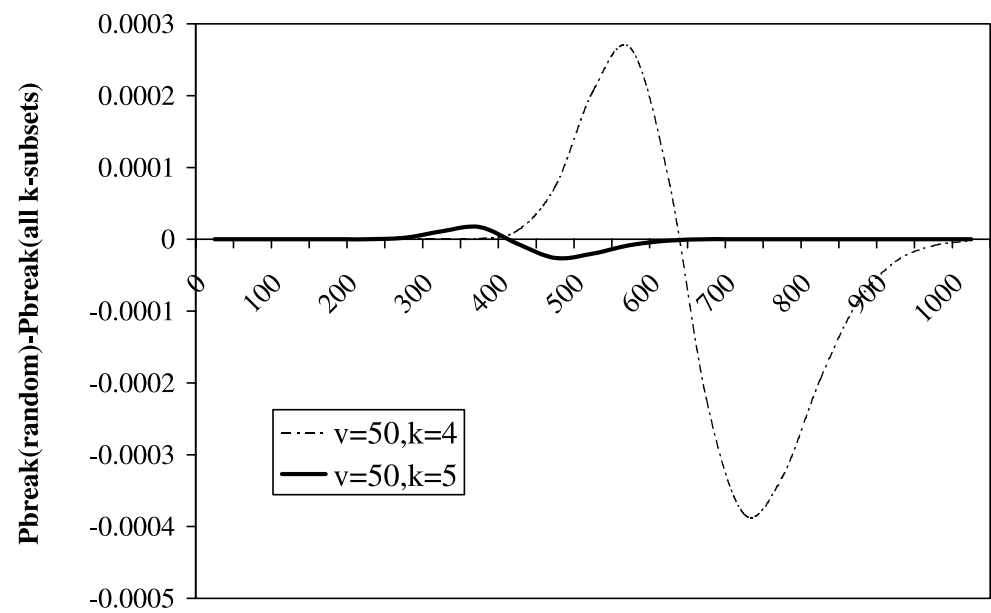

Number of Compromised Node

Fig. 3. Difference between $P_{b r e a k}$ of all $k$-subsets and random scheme

\section{Conclusion}

In this paper, we introduced a generalized $D \times S$ key pre-distribution scheme for sensor networks. We deduced conditions of the set system used in the scheme that can provide optimal connectivity and resilience to the sensor network. Based on the result we analyzed some existing key pre-distribution schemes and evaluated their strength and weakness. Then we proposed 2 specific schemes and their constructions that can achieve optimal connectivity and resilience.

This paper is focused on optimal connectivity and resilience of the key distribution scheme. Another important property of the schemes is scalability. In real implementation, the scale of the sensor networks often impacts connectivity and resilience. In the future research, we are going to focus on scalability and its relationship with connectivity and resilience, and look for optimal schemes.

\section{Acknowledgements}

The authors wish to thank D.R. Stinson for informing us the construction in Theorem 2. R. Wei's research is supported by NSERC grant 239135-01.

\section{References}

1. Wireless Integrated Network Sensors, University of California, http://www. janet.ucla.edu/WINS. 
2. I.F. Akyildiz, W. Su, Y. Sankarasugramaniam and E. Cayirci, A survey on sensor networks, IEEE Communications Magzine, 40(2002), 102-114.

3. R. Blom, An optimal class of symmetric key generation systems, Advances in Cryptology: EUROCRYP 84 (T. Beth, N. Cot and I. Ingemarsson, eds.) LNCS 209 (1985), 335-338.

4. H. Chan, A. Perrig and D. Song, Random key predistribution schemes for sensor networks, IEEE Sumposium on Research in Security and Privacy, (2003), 197-213.

5. C. J. Colbourn and J.H. Dinitz, The CRC Handbook of Combinatorial Designs, CRC Press, 1996.

6. W. Du, J. Deng, Y. S. Han and P. K. Varshney, A pairwise key pre-distribution scheme for wireless sensor networks, Proc. of the 10th ACM conf. on Computer and communications Security, (2003), 42-51.

7. L. Eschenauer and V. D. Gligor, A key-management scheme for distributed sensor networks, Proc. of the 9th ACM conf. on Computer and communications Security, (2002), 41-47.

8. Erdös and Rényi, On random graphs I. Publ.Math. Debrecen, 6(1959), 290-297.

9. J.M. Kahn, R.H. Katz and K.S.J. Pister, Next century challenges: Mobile networking for smart dust, In: Proceedings of the 5th Annual ACM/IEEE Internation Conference on Mobile Computing and Networking, (1999), 483-492.

10. D. Liu and P. Ning, Establishing pairwise keys in distributed sensor networks, Proc. of the 10th ACM conf. on Computer and communications Security, (2003), 52-61.

11. D.R. Stinson, Combinatorial Designs: Constructions and Analysis, Springer, New York, 2003. 